\title{
Transmembrane Kinases are essential for plant development
}

Qiang $\mathrm{Li}^{1,2,3, \#}$, Jie Yang ${ }^{1,2,3, \#}$, Yi Zhang ${ }^{1,2,3}$, Fen Wang ${ }^{1}$, Mingzeng Chang ${ }^{1,2,3}$, Tongda $\mathrm{Xu}^{1,3}$, Shui Wang ${ }^{1,4, *}$ and Jun $\mathrm{He}^{1,3, *}$

${ }^{1}$ Shanghai Centre for Plant Stress Biology, Centre for Excellence in Molecular Plant Sciences, Shanghai Institutes for Biological Sciences, Chinese Academy of Sciences, Shanghai, P. R. China.

${ }^{2}$ University of Chinese Academy Sciences, Beijing, P. R. China.

${ }^{3}$ FAFU-UCR Joint Centre, Horticulture and Metabolic Biology Centre, Haixia Institute of Science and Technology. Fujian Agriculture and Forestry University, Fuzhou, Fujian, P. R. China.

${ }^{4}$ College of Life and Environmental Sciences, Shanghai Normal University, Shanghai, P. R. China.

\# These authors contributed equally to this work.

*Correspondence:0000ptd004@fafu.edu.cn

\section{One-sentence summary:}

Multiple tmk mutants analysis illustrated the key roles of TMKs in plant development.

\section{Footnotes}

Financial source: This work was supported by National Natural Science Foundation of China (Grant 31470359) to J. H.

*Corresponding author: Jun He (0000ptd004@fafu.edu.cn) and S. W. (shuiwang@ shnu.edu.cn) 


\section{SUMMARY}

Transmembrane kinase family proteins (TMKs) have been implicated in regulating both auxin signaling and plant development. To obtain insights of the potential TMKs' function in plant development, we regenerated new full sets of tmk mutants, and discovered many new phenotypes, such as defective organogenesis, smaller rosette leaves, fertility defects and selfing population defects in different combination of $t m k$ mutants. Taken together, our results demonstrated that TMKs participated in multiple aspects of plant development, which provided a great reference for any future research.

Keywords: Arabidopsis thaliana, transmembrane kinase family proteins (TMKs), plant development 


\section{INTRODUCTION}

Plant growth and development are regulated by different triggers like phytohormones or environments (Santner and Estelle, 2009 , Csaba, 2015). Auxin, as the first identified phytohormone, is involved in nearly all aspects of plant lifecycle processes (Mockaitis and Estelle, 2008). The previous study suggested that auxin is required in the apical-basal axis formation in egg cells before fertilization (Mansfield et al., 1991 , Laux and Jurgens, 1997), in the root apical meristem and shoot apical meristem maintenance (Sassi and Traas, 2015 , Azizi et al., 2015 , Della Rovere et al., 2013), in cotyledon initiation during the embryogenesis transition stage (Moller and Weijers, 2009) and in the formation of the lateral roots (Benkova et al., 2003, Dubrovsky et al., 2008).

Auxin signal was reported to be recognized and transduced by its binding protein the cell surface based (Auxin binding protein 1)ABP1 (Hertel et al., 1972 , Dharmasiri et al., 2005 , del Pozo et al., 2006 , Jurado et al., 2008). ABP1 specifically binds with auxin with high affinity, but its developmental role is still unclear (Gao et al., 2015). TMK1 is identified as the docking protein of ABP1 at the cell surface, which mediates auxin activation of Rho GTPase signaling pathway (Klambt, 1990, Robert et al., 2010, Xu et al., 2010). TMK proteins are members of a subfamily of Leucine-rich repeat RLK family, which contains four family members. All four TMKs have the conserved LRR motif, the transmembrane region and the cytoplasmic kinase domain (Dai et al., 2013). The first identified member of them is TMK1 and it was characterized in the course of a chromosome walk to the ethylene response locus, and further proved as a kinase for serine and threonine by in vitro kinase assay (Chang et al., 1992). The kinase exhibited greater autophosphorylating activity with $\mathrm{Mn}^{2+}$ than with $\mathrm{Mg}^{2+}($ Schaller and Bleecker, 1993). Recent work demonstrates that auxin accumulation at the concave side of the apical hook stimulates TMK1 cleavage followed by its nuclear translocation to regulate gene transcription via stabilizing IAA32 and IAA34, two non-canonical Aux/IAA proteins lacking domain II which is required for interaction with the TIR receptors (Cao et al., 2019). TMK4 was first cloned as BAK1-Associating Receptor-Like Kinase 1 (BARK1) and reported to regulate BR-mediated plant development including lateral roots via auxin regulation (Kim et al., 2013). Moreover, TMK4 was reported to interact with and phosphorylates TAA1 at Thr101 site, which negatively regulates auxin biosynthesis that is essential for the regulation of the local 
auxin concentration in plants (Wang et al., 2020). And also TMK1/TMK4-mediated phosphorylation and activation of MKK4/MKK5, followed by the activation of MPK3/MPK6, are involved in cell division during lateral root (LR) development (Huang et al., 2019). All these indicate the multiple functions of TMKs at least in auxin signaling.

The T-DNA insertion mutants of these four TMKs were isolated previously (Dai et al., 2013 , Schwachtje et al., 2012). And no obvious developmental phenotype was observed in the tmk single mutants. But in the tmk1/4, tmk1/3/4 and tmk1/2/3/4 mutants, the development of the root, hypocotyl and stamen filament was strongly reduced due to both cell division and elongation defects (Dai et al., 2013). The tmk1/2/3/4 quadruple mutant is sterile (Schwachtje et al., 2012, Dai et al., 2013). Moreover, the tmk mutants showed less sensitivity to exogenous auxin treatment in primary root growth, lateral root initiation and pavement cell interdigitation (Dai et al., 2013, Xu et al., 2014). The comprehensive phenotypes in multiple tmk mutants suggest the essential roles of TMKs in plant development, which needs to be further carefully investigated. Here, by regenerating different combinations of multiple tmk mutants in pure Col-0 background, we further extended our understanding of the TMKs function in regulating plant development, including vegetative development and fertilization processes. Taken together, our results demonstrated that TMKs modulate organ developmental programming and adaptive growth to the environmental stimulus, which is essential for Arabidopsis development. 


\section{RESULTS}

\section{Novel tmk T-DNA insertion mutants with pure Col-0 background}

The phenotypes of Arabidopsis varied excessively among different ecotypes (Schwachtje et al., 2012). Although the important roles of TMKs in auxin signaling, previous tmks with mixed ecotypes might be debated in the whole TMK and auxin research society. There are total 4 members of TMKs subfamily which locate at different chromosomes in Arabidopsis genome (Figure 1A). To study the functions of these 4 members of TMKs subfamily in Arabidopsis, we collected and identified each TMKs single knock-out T-DNA mutant in Col-0 background from publicly available resources. The tmk1-/-(tmkl) single mutant was the same as before because it is Col-0 background already (Dai et al., 2013). Novel tmk2, tmk3 and tmk4 knockout mutants were identified and the T-DNA insertion is located at positions $+1550 \mathrm{bp},+2106$ bp, $+137 \mathrm{bp},+2559 \mathrm{bp}$ relative to the start codons of their corresponding genes, respectively (Figure 1B). The expression of each $T M K$ in the corresponding individual tmk single mutant was confirmed by genotyping and quantitative real-time RT-PCR (qRT-PCR) (Figure1D and 1E). To generate novel tmk mutant combinations, the $t m k 1$ single mutant was crossed with the novel $t m k 2-/-(t m k 2)$ single mutant and, meanwhile, the novel tmk3-/-(tmk3) single mutant was crossed with the novel tmk4-/-(tmk4) single mutant, respectively. The F1 generation lines tmk1+/-tmk2+/- and tmk3+/-tmk4+/- were then crossed with each other. In the next generation, the heterozygous line tmk1+/-tmk2+/-tmk3+/-tmk4+/- was obtained by genotyping. The seeds of this line were collected to further screen candidates for double, triple, and quadruple tmk mutants. Finally, different combinations of novel tmk mutants, including 4 tmk single mutants, 6 tmk double mutants, 4 tmk triple mutants and one tmk quadruple mutant were obtained.

\section{TMKs are required for vegetative development in Arabidopsis}

We further examined carefully whether there are any abnormal vegetative developmental phenotypes in these new tmk mutants. We focused on 4 developmental characters, the biggest rosette leaf (BRL) at the juvenile stage and the primary inflorescence height (PIH) at the adult stage, the primary root length (PRL) and etiolated hypocotyl length. All tmk single mutants showed no obvious defects (Figure 2), except tmk4 single mutant which exhibited obvious growth defects in BRL (around 22.3\% reduction) (Figure 2A and 2B) and PRL (around 26\% reduction) in PRL comparing to Col-0 wild type (Figure 2E and 2F). In contrast, 
severe developmental defects were observed in tmk1-/-tmk4-/- (tmk1/4) double, tmk1-/-tmk2-/-tmk4-/(tmkl/2/4) and tmk1-/-tmk3-/-tmk4-/- (tmk1/3/4) triple and tmk1-/-tmk2-/-tmk3-/-tmk4-/- (tmk1/2/3/4) quadruple mutants. The BRL in tmkl/4 double mutant and tmkl/2/3/4 quadruple mutant was around 38\% and $21 \%$ of the Col-0, respectively (Figure 2A and 2B). The PIH in tmk $1 / 4$ double mutants was $59 \%$ of the Col-0, and only $12 \%$ that of the Col-0 in the tmk1/2/3/4 quadruple mutants (Figure 2C and 2D). The tmk1/2/4 and tmk1/3/4 triple mutants displayed slightly severe phenotype at BRL and PIH compared with tmkl/4 double mutants (Figure 2A, 2B, 2C, and 2D). The PRL in tmkl/4, tmk1/2/4, and tmkl/3/4 mutants was around 1/3 that of the Col-0, whereas about $1 / 6$ that of the Col-0 in the tmk1/2/3/4 quadruple mutants was observed at the early stage of seedling development (Figure 2E and 2F). For the hypocotyl length, tmk1/2/3/4 was shortest (how much reduction) followed by tmkl/4 (70\% reduction) compared to Col-0. Furthermore, we confirmed that the tmkl/4 complementation line (complemented by native promoter driven $g T M K 1$, see experimental procedures) rescued the hypocotyl phenotype (data not shown). Taken together, tmk1//2/3/4 shows the most severe growth defect phenotypes followed by tmk1/2/4 or tmkl/3/4 and $t m k 1 / 4$. Therefore, we concentrated on these mutants, especially tmkl/4 and $t m k 1 / 2 / 3 / 4$, for further study.

In previous $t m k 1+/$-tmk2-/-tmk3-/-tmk4-/- $($ tmkl+/-tmk2/3/4) heterozygous lines, the single cotyledon phenotype was observed (Xu et al., 2014). Interestingly, besides single cotyledon, we also observed triple and swollen cotyledons in our novel tmk1-/-tmk2-/-tmk3-/-tmk4+/- (tmk1/2/3;tmk4+/-) mutants (Figure 4A and 1B). The tmk1/2/3 triple mutants displayed cotyledon-shaping defects as well, although with an even lower frequency (about 0.26\%) (Figure 4C). Furthermore, the rootless phenotype coupled with triple cotyledons in novel tmk1/2/3/4 quadruple mutants were also identified (Figure 4B).

Taken together, our data suggested that functions of TMKs in Arabidopsis vegetative growth from seedling stage to adult stage are essential and largely overlapped. In addition, roles of TMK4 were irreplaceable by the other 3 TMKs during Arabidopsis vegetative growth. Since tmk1//2/3/4 showed the most severe growth defect phenotypes followed by tmkl/2/4 or tmkl/3/4 and tmkl/4. Therefore, we focused on these mutants, especially tmkl/4 and $t m k 1 / 2 / 3 / 4$, for further study.

\section{TMKs are required for Arabidopsis reproductive growth}


Next, we examined whether there are any abnormal phenotypes at reproductive growth stages in these new tmk mutants. We found that the tmk1/2/3/4 mutant showed the shortest inflorescence shoot and silique phenotype followed by tmkl/4 mutant but no obvious phenotypes were observed in tmk single mutants (Figure $3 \mathrm{~A}$ and $3 \mathrm{~B}$ ). In agreement with the reduced silique length, the mature seed number was dramatically decreased, in $t m k 1 / 4, t m k 1 / 2 / 4$, and $t m k 2 / 3 / 4$ mutants, to $18.8 \%, 14.5 \%$, and $41.6 \%$ that of wild type, respectively (Figure 3C). Strikingly, there are no matured seeds produced in siliques of tmkl/3/4 and tmk1/2/3/4 mutants (Figure 3C), which implied an essential role of TMKs in the fertilization process or embryo development. To further investigate this hypothesis, whole flower organ of tmks mutants were observed firstly, among which $t m k 1 / 2 / 3 / 4$ displays obvious size reduction followed by $t m k 1 / 4$ combinations ( $t m k 1 / 3 / 4, t m k 1 / 2 / 4$, and $t m k 1 / 4)$ and $t m k 2 / 3 / 4$ (Figure 3D). We further split out the flowers of these mutants and found the petal, sepal, pistil, and stamen are all reduced in size/length, especially in the tmk1/2/3/4 quadruple mutant, although no shapes and numbers seem to be defective (Figure 3 E-G). When peeling off the silique, we found there are totally aborted seeds that seem to arise from fertility in the aforementioned tmk mutants (Figure $3 \mathrm{H}$ ). From this part of the view, tmk1/2/3/4 quadruple mutants were completely infertile, followed by tmkl/4 and tmk2/3/4 mutants, whereas no fertility defects in single $t m k$ mutants (Figure $3 \mathrm{H}$ ). Occasionally, it was interesting to identify that all the stamens in novel tmk1/2/3/4 quadruple mutants were shorter than the stigma (Figure $3 \mathrm{~F}$ and $3 \mathrm{G}$ ). Thus, to study the infertility phenotype of tmk mutants caused by the stamen or the pistil, the pollen of the tmk1/2/3/4 quadruple mutant was smeared on the stigma of Col-0, and then ( 3 weeks later) the siliques were split out and only 3 to 5 mature seeds could be obtained in one silique on average (Figure 3I, the left panel, indicated by red arrows) suggesting the anther from tmk1/2/3/4 quadruple mutant has extremely severe fertility defects. No mature seeds could be observed in the siliques no matter the smeared pollen was from Col-0 or the novel tmk1/2/3/4 quadruple mutant (Figure 3I, the middle and right panels, indicated by red arrows) suggesting the pistil from tmk1/2/3/4 loses the fertility thoroughly. Based on these results, the sterility of $t m k 1 / 2 / 3 / 4$ quadruple mutants might be caused by defects in both stamen elongation and pistil development. Thus, TMKs might play previously unknown roles in the regulation of stamen elongation, ovule and pollen development, and seed maturation. In addition, according to the very low ratio $(7.69 \%+$ $0.38 \%=8.07 \%$, which is far less than the theoretical ratio $25 \%$; Figure $4 \mathrm{~B}$ ) of homozygous $t m k 1 / 2 / 3$; 
$T M K 4-/$ - from the $t m k 1 / 2 / 3 ; T M K 4+/$ - parental generation, it is noteworthy that TMKs must also play a role in embryo development.

\section{DISCUSSIONS}

Here, our findings present several important implications. First, our results confirmed functionally overlapping roles of the TMKs and pointed out the unique and irreplaceable status of TMK4 among four TMKs. Second, phenotype characterization of novel tmk mutant combinations expanded the roles of TMKs from fundamental growth to adapted growth in Arabidopsis. Third, our data provided genetic evidence more accurately and comprehensively for future studies of TMKs family. All these findings here render roles of TMKs to more diverse and more general aspects of Arabidopsis developments. Here we applied multiple model systems at different tissue/organ levels to investigate roles of TMKs in Arabidopsis development. Future studies are required for deciphering the mechanisms of TMKs mediated multiple developmental processes at the molecular and biochemical cellular levels.

Characterizations of novel tmk mutants reveal TMKs orchestrate many fundamental aspects of

\section{Arabidopsis growth and development}

In this paper, we generated and comprehensively studied novel tmk combination mutants with pure ecotype background. We characterized the loss function of TMKs as dampens both vegetative growth and reproductive growth including primary roots, hypocotyls, rosette leaves, inflorescence, flower organs, siliques, and seeds maturation. These results are greatly consistent with Bleecker's group, who showed severe growth and development defects of tmk1;tmk2;tmk3;tmk4 (they generated) quadruple mutant in roots, hypocotyls, leaves, and stamen filaments caused by hampered cell growth and proliferation, although the T-DNA insertion mutants of the four TMKs contains ecotype mixture (Dai et al., 2013). Similarly, among all the novel tmk mutant combinations, the novel tmkl/4 double, tmkl/2/4 and $t m k 1 / 3 / 4$ triple and tmk1/2/3/4 quadruple mutants displayed especially severe defects in almost all examined development processes of diverse Arabidopsis organs, suggesting the outstanding roles of TMK1 and TMK4 among the four functional redundant TMKs in Arabidopsis. Unlike the previous tmk4 single mutant, here we discovered obvious defects of novel tmk 4 single mutant in primary rosette leaf expansion 
(Figure 2A and 2B) and root elongation (Figure 2E and 2F). Novel tmk1/2/3/4 displayed significantly enhanced defects in both primary inflorescence and pavement cells (PCs) interdigitation (Figure 2C and 2D) as compared to the previous one (Xu et al., 2014 , Dai et al., 2013). These results emphasized the unique role of TMK4 among four TMKs in the vegetative growth of Arabidopsis and indicated that the previous mutants with mixed ecotypes might cover the phenotype defects both at the organic level and cellular level during Arabidopsis vegetative growth. In the reproductive growth of Arabidopsis, the phenotype defects covered by the ecotype mixture seem to be much stronger, although fertility in most novel tmk mutants was similar to the Col-0 and the novel tmk1/2/3/4 quadruple mutants were also completely infertile like previous ones (Dai et al., 2013) (Figure 3). More severe infertility showed up in novel tmkl/4 double mutants as compared to previous tmkl/4 mutants: only $18.8 \%$ seeds per silique as compared to wild type were matured successfully in the silique of novel tmkl/4 double mutants, rather than $30.7 \%$ that in the previous tmkl/4 double mutants (Figure 3C; Dai et al., 2013). What's more, the novel tmk2/3/4 triple mutant also displayed severe infertility while the previous one didn't show visible fertility defects. Finally, both severe fertility defects of stamen and completely fertility of pistil result in the infertility phenotype of $t m k 1 / 2 / 3 / 4$ mutant (Figure 3I). More interestingly, both the low homozygous ratio in progenies of $t m k 1 / 2 / 3 ; t m k 4+/-$ and aborted seeds growth observed in tmkl/4 and tmk2/3/4 hint the embryo development defects also exist as confirmed in a previous paper by using previous tmk1/2/3/4 mutant (Xu et al., 2014). Moreover, the ratio of abnormal cotyledon morphology in progenies of tmk1/2/3; tmk4+/- (3.2\%) was lower than that of tmkl-/+tmk234 (17.1\%) (Figure 4C) (Xu et al., 2014). It is likely that the embryo development and phenotype in the novel tmk quadruple mutants must be easily affected by growth conditions because the homozygous tmk1/2/3/4 ratio is $8.08 \%$ (Figure $4 \mathrm{C}$ ), much lower than the $25 \%$ separation ratio, and also we can't exclude the reason from ecotype mixture. These findings expanded the roles of TMKs to more detailed and deeper aspects of reproductive growth in Arabidopsis. Apart from tmk1/2/3/4 quadruple mutant, $\mathrm{PCs}$ of all the other tmk mutant combinations are firstly examined here. We found that novel tmk1/2/3/4 quadruple mutant displayed similar PCs interdigitation defects as reported (Figure 5) (Xu et al., 2014). Taken together, although the tmk mutants possess similar growth and development defects which match that of the previous one to a large content, several phenotypes observed here differed from previous ones which still cannot exclude the ecotype 
mixture problems. Therefore, the new mutants could be better research materials for future study.

\section{Function specificity of each TMK}

By using promoter:GUS reporter system and qRT-PCR analyses, TMK1, TMK3, and TMK4 were demonstrated to be widely expressed in all organs tested including roots, stems, leaves, flowers, and siliques, whereas $T M K 2$ expression was in trace amounts only detected in flowers and siliques of wild type Arabidopsis (Dai et al., 2013). We confirmed the knock-out tmkl and tmk4 mutants through checking transcripts abundance by qRT-PCR (Figure 1). The relatively more important roles of TMK1 and TMK4 may contribute to the more prevalent expression patterns among organs in Arabidopsis. Substantial serious defects of novel tmkl/4 and novel tmkl/2/3/4 mutants are also consistent with the particular expression pattern of each TMK. TMK4 itself also plays roles in growth relate phenotypes such as rosette leaf size and primary root elongation (Figure $1 \mathrm{~A}, 1 \mathrm{~B}, 1 \mathrm{E}$, and $1 \mathrm{~F}$ ) suggesting its dominant and specific function in these tissues. To our surprise, we also discovered the novel tmk2/3/4 triple mutant showed reduced fertility (Figure 3G), which indicates that the roles of TMK2, TMK3, and TMK4 in controlling sexual growth. In the future, the expression pattern data with higher resolution should be obtained and examined carefully to separate the function of each TMK. What's more, the expression level of each TMK in different tmk mutant combinations should be investigated simultaneously. And it's worthy to note that apart from the T-DNA insertion lines, the other TMKs knock down or knock out lines are still necessarily going to be constructed by other technologies. As described above, TMK4 is also named as BRI1-ASSOCIATED RECEPTOR KINASE1 (BAK1)-associating receptor-like kinase 1 due to its binding to BAK1 (Kim et al., 2013). Further study in auxin-brassinosteroid crosstalk may consider TMK4 as the possible link component. 


\section{EXPERIMENTAL PROCEDURES}

\section{Plant Materials and Growth Condition}

Arabidopsis thaliana seeds were surface-sterilized in $70 \%$ (v/v) ethanol for 10 minutes, and rinsed with sterile water 3 to 5 times. After stratified at $4^{\circ} \mathrm{C}$ for 2 to 5 days in the dark, seeds were placed on $1 / 2$ Murashige and Skoog (MS) medium supplemented with 1\% (w/v) sucrose and $0.8 \%(\mathrm{w} / \mathrm{v})$ agar for 2 weeks in a growth chamber and then 2-week-old seedlings were transferred to soil for further growth in greenhouse. The growth condition of both the growth chamber and the greenhouse is at $22^{\circ} \mathrm{C}$ with a $16-\mathrm{h}$ light/8-h dark cycle at $75 \mu \mathrm{mol} \mathrm{m} \mathrm{m}^{-2} \mathrm{~s}^{-1}$ and $65 \%$ humidity.

The T-DNA insertion lines of both tmk1-/- (SALK_016360) and tmk2-/- (SAIL_1242_H07) used here are previously reported single mutants with pure Columbia-0 (Col-0) ecotype. The Col-0 background tmk3-/- (SALK_129759) and tmk4-/-(GABI_191D02) single mutants were obtained from the Nottingham Arabidopsis Stock Centre (NASC; http://Arabidopsis.info). The transgenic lines DR5p::GUS and DR5p::GFP with Col-0 ecotype were kindly supported by Shingo Nagawa. DR5p::GFP/GUS with tmkl/4 background (DR5p::GFP/tmk1/4) was generated from DR5p::GFP/GUS crossing with tmk1/4 mutant. For three tmkl/4 complementation lines driven by TMK1 promoter, tmk1/4:gTMK1-2, tmk1/4:gTMK1-4, and tmk1/4:gTMK1-5, please see the complementation assay.

\section{Identification of T-DNA Insertion Mutants}

According to previous reports, genotype specific PCR was used for identifying T-DNA insertion sites of these Col-0 background tmk mutants. Generally, LB and RP primer set was used to identify T-DNA insertion while LP and RP primer set was used to confirm homozygous insertion. For the primer used, For LB primers, LBb1.3, LB1, and GABI-08409-LB primer were used for "SALK", "SAIL" and "GABI" mutants, respectively. The corresponding LP and RP primer sets were designed at the website (http://signal.salk.edu/tdnaprimers.2.html). For primer sequence, please see Supplemental Table 1. Procedure for PCR amplification by using ExTaq polymerase (Takara): $94^{\circ} \mathrm{C} 2 \mathrm{~min} ; 35$ cycles of $94^{\circ} \mathrm{C}$ $20 \mathrm{~s}, 62^{\circ} \mathrm{C} 30 \mathrm{~s}$, and $72^{\circ} \mathrm{C} 45 \mathrm{~s} ; 72^{\circ} \mathrm{C} 5 \mathrm{~min}$.

\section{Complementation Assay}


To generate the tmk1/4:gTMK1 complementation line, $3.5 \mathrm{~kb} T M K 1$ promoter driven genomic sequence plus $1 \mathrm{~kb}$ 3'-UTR of TMKl were PCR amplified and inserted into pDONR-Zeo vector to construct pDONR-gTMK1, FLAG tag was inserted into 3'-end of gTMK1 (pDONR-gTMK1-FLAG) and subsequently, gTMK1-FLAG was transferred into pGWB501 destination vector by LR reaction. The recombinant vector gTMK1-FLAG-pGWB501 was introduced into the tmkl/4 mutant plants via Agrobacterium tumefaciens-mediated transformation (Clough and Bent, 1998). Transgenic plants were screened by $1 / 2$ MS plates (1\% sucrose) with $25 \mu \mathrm{g} / \mathrm{ml}$ of Hygromycin B. Finally, three T3 homozygous tmk1/4 complementation lines driven by TMK1 promoter, tmk1/4:gTMK1-2, tmk1/4:gTMK1-4, and tmk1/4:gTMK1-5, were obtained (Figure 2C: line 2, line 4, and line 5).

\section{RNA Isolation, cDNA Synthesis and Quantitative Real-time RT-PCR (qRT-PCR)}

5-dag (day after germination)-old seedlings of tmk mutants grown in the growth chamber were frozen and ground by liquid nitrogen. RNA was isolated by Trizol RNA isolation kit (Invitrogen). After RNA isolation, reverse transcription was performed using cDNA synthesis superMix (TransGen Biotech). Actually, total $500 \mathrm{ng}$ to $1 \mu \mathrm{g}$ RNA was added in a total reaction volume of $20 \mu \mathrm{L}$. Reverse transcribed cDNA was diluted to 10 times with $\mathrm{ddH}_{2} \mathrm{O}$ before quantitative real-time RT-PCR. For qRT-PCR, $2.5 \mu \mathrm{M}$ forward and reverse primer set, $3 \mu \mathrm{L}$ cDNA template and $4 \mu \mathrm{L}$ iQ SYBR green supermix (Bio-Rad) in a total reaction volume of $12 \mu \mathrm{L}$. The reaction was detected by using CFX96 ${ }^{\mathrm{TM}}$ Real-Time System (Bio-Rad). TUB4 (AT5G44340) was used as a control gene. For primer used to detect the TMK gene expression level, please see Supplemental Table 1.

\section{Phenotypic Analysis}

Actually, "10+14" plants (10-dag-old seedlings grown in a growth chamber, transferred in soil and grown in the greenhouse for another 14 days) were used for the analysis of the diameter of the biggest rosette leaf (BRL). For measurement of the primary inflorescence height $(\mathrm{PIH})$, “10+35" plants were used. For detection of the primary root length, 4-dag-old seedlings grown in a growth chamber were measured. For quantification of pavement cell bulge/interdigitation, single layer scanned images were overlapped by LAS AF Lite software and then analyzed by Photoshop CS6 software. A stereomicroscope 
(Leica S8 APO and Leica DM6000B) was used to observe the sexual growth phenotype including flower, silique, stigma, and stamen. ImageJ was used to process the length measurement.

\section{Propidium Iodide (PI) Staining}

For, PI staining, $10 \mu \mathrm{g} / \mathrm{mL}$ Propidium iodide (Sigma) was used to stain pavement cells for two days.

\section{Microscopy}

For PI signal detection, a Leica TCS SP8 SMD microscope system was used. The argon laser was switched to $488 \mathrm{~nm}$ to detect GFP signal between $500 \mathrm{~nm}$ and $550 \mathrm{~nm}$. The argon power was set at $20 \%$. While $561 \mathrm{~nm}$ argon laser with power of $5 \%$ was used to detect PI signal between $560 \mathrm{~nm}$ and $580 \mathrm{~nm}$.

\section{ACKNOWLEDGMENTS}

No conflict of interest was declared. We would like to thank ABRC for providing seeds of SALK_016360 and SAIL_1242_H07, and to thank the NASC for providing seeds of SALK_129759 and GABI_191D02. This study was supported by National Natural Science Foundation of China (Grant 31470359) to J. H.

\section{ACCESSION NUMBER}

Sequence data from this study can be found in the Arabidopsis Genome Initiative or GenBank/EMBL databases under the following accession numbers TMK1 (AT1G66150), TMK2 (AT1G24650), TMK3 (AT2G01820), TMK4 (AT3G23750).

\section{SUPPORTING INFORMATION}

Supplemental Table 1. List of primers used in this study. 


\section{FIGURE LEGENDS}

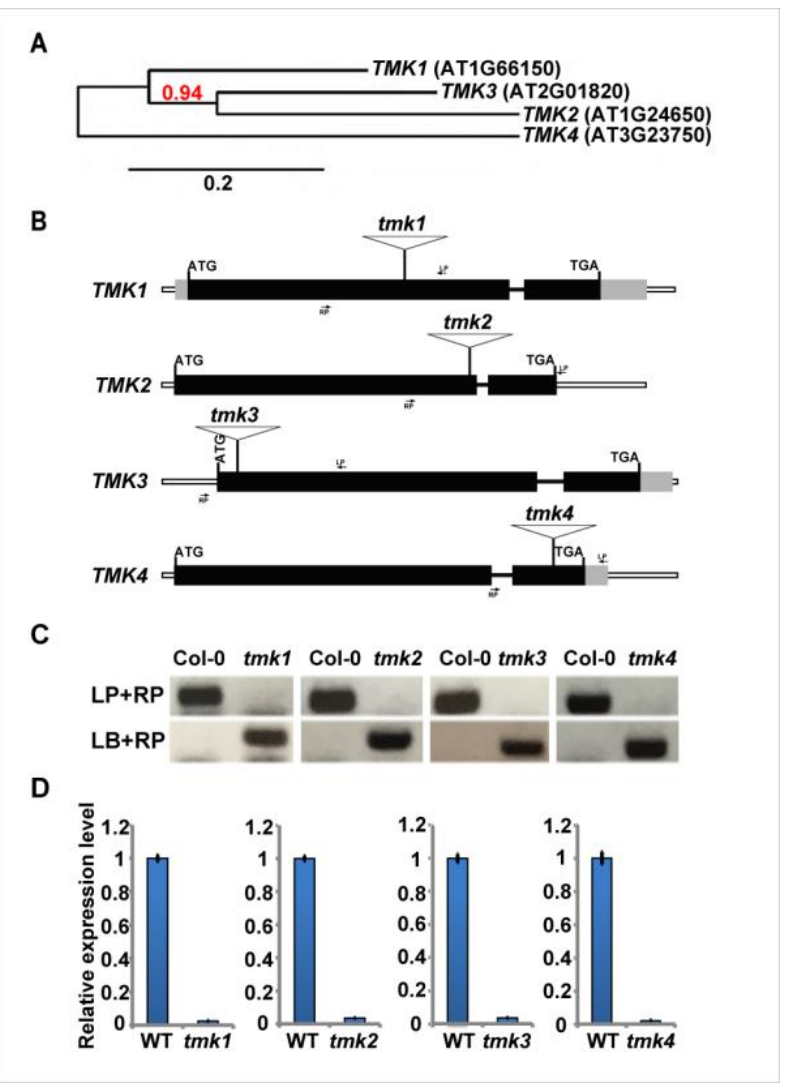

Figure 1. Generation of novel mutant combinations among TMKs.

(A) The phylogenetic tree of TMKs in Arabidopsis genome predicted by MEGA 5.

(B) Identification of the T-DNA insertion sites of the tmk mutants. The black boxes, gray boxes, lines, and long boxes indicate the exons, untranslated regions, introns, and interval regions, respectively The locations of LB and RP primer pair are indicated by arrows.

(C) Genotyping of 4 tmk single mutants. No bands show up in $t m k 1$, novel $t m k 2$, tmk3, and $t m k 4$ single mutants as compared to Col-0 in the gene specific PCR groups (the upper panel). No bands present in Col-0, but a band is shown in $t m k 1$ and novel $t m k 2, t m k 3$, and $t m k 4$ single mutant in the T-DNA specific PCR group (the lower panel).

(D) Gene expression of $T M K s$ in their corresponding mutants by using qRT-PCR. The transcripts of $T M K 1$, $T M K 2, T M K 3$, and $T M K 4$ are almost undetectable in the $t m k 1$ and novel $t m k 2, t m k 3, t m k 4$ single mutants, respectively. $U B Q 1$ was applied as an internal control. Error bars are SD. 
A

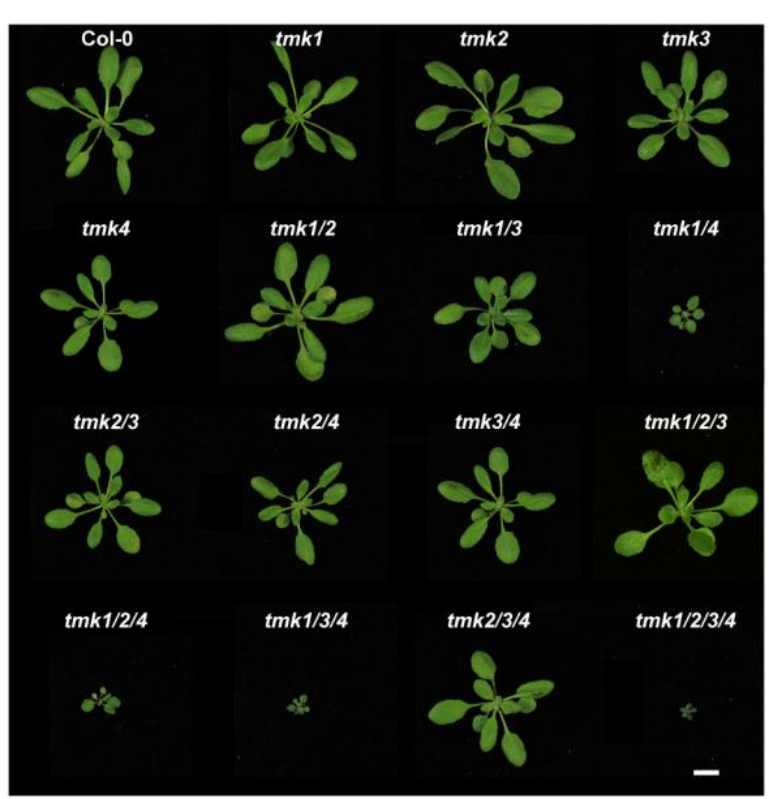

B

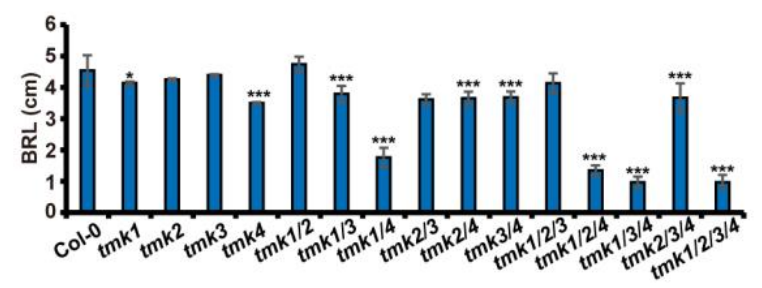

E

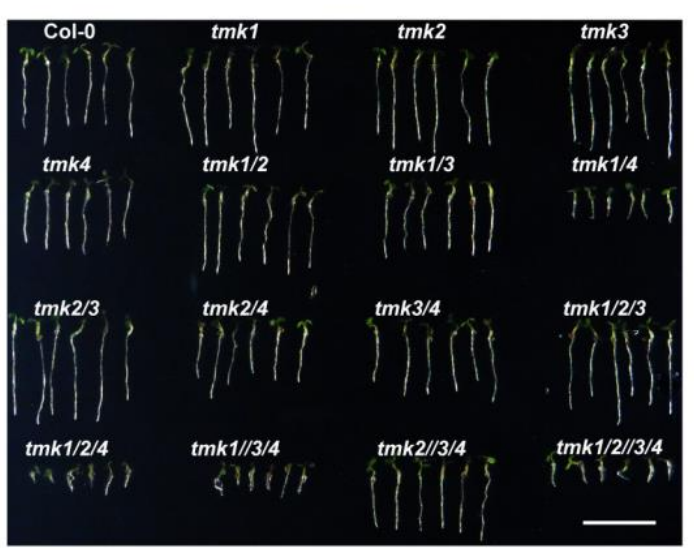

C

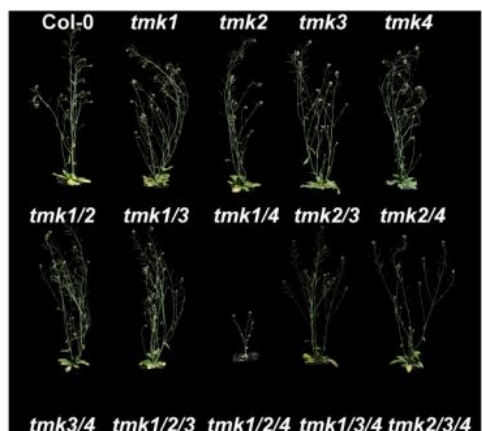

tmk3/4 tmk1/2/3 tmk1/2/4 tmk1/3/4 tmk2/3/4

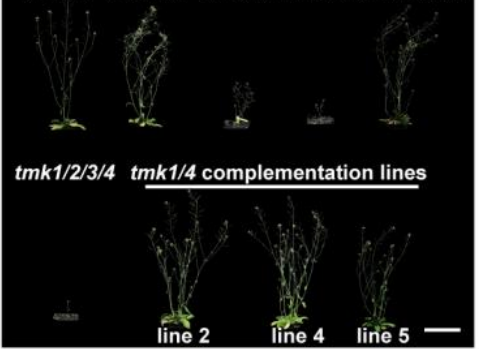

D

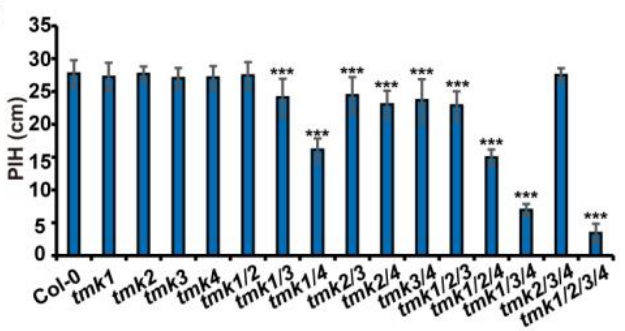

$\mathbf{F}$

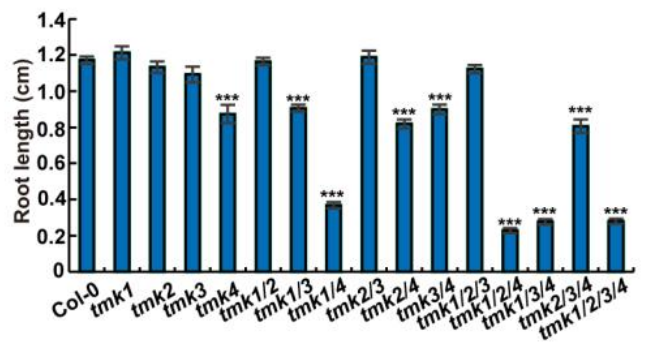

Figure 2. The phenotype characterization of the novel tmk mutants and the complementation lines.

(A) The 24-dag-old (grown in a growth chamber for 10 days and transferred to soil and grown in the greenhouse for another 14 days) plants of novel tmk mutants and Col-0. Bar, $10 \mathrm{~cm}$.

(B) Quantitative analysis of rosette leaf size of novel tmk mutants.

(C) The 45-dag-old (10-dag-old seedlings transferred to soil and grown for another 35 days) plants of novel tmk mutants, Col-0, and the tmk1/4 complementation lines (tmk1/4:gTMK1, see experimental procedures). 
Bar, $5 \mathrm{~cm}$.

(D) Quantitative analysis of primary inflorescence height (PIH). Shown are average \pm SD ( $n=30)$.

(E) The 4-dag-old seedlings of novel tmk mutants and the Col-0.

(F) Quantification of root length. Shown are average \pm SD $(n=30)$.

Note: t-test for comparison between tmk mutants and Col-0. Error bars denote \pm SD. “*”, “**”, and “***” indicate $\mathrm{p}<0.05, \mathrm{p}<0.01$, and $\mathrm{p}<0.001$, respectively. 
A
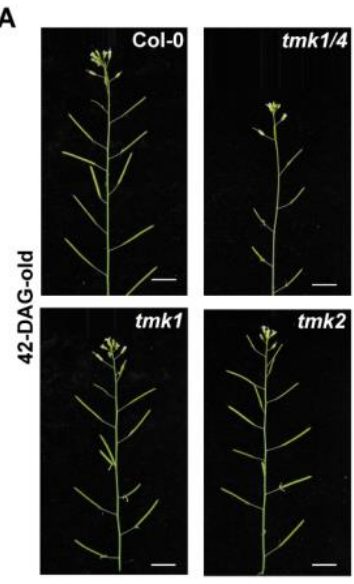

D

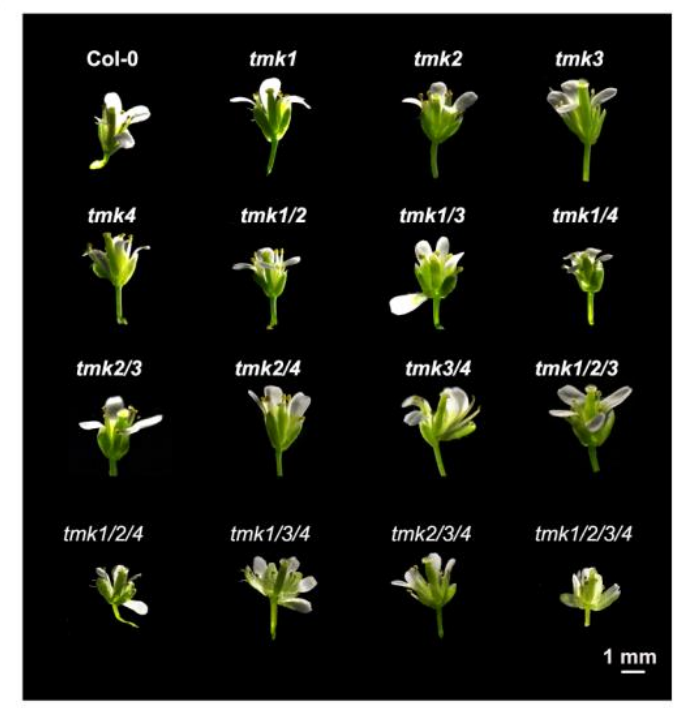

H

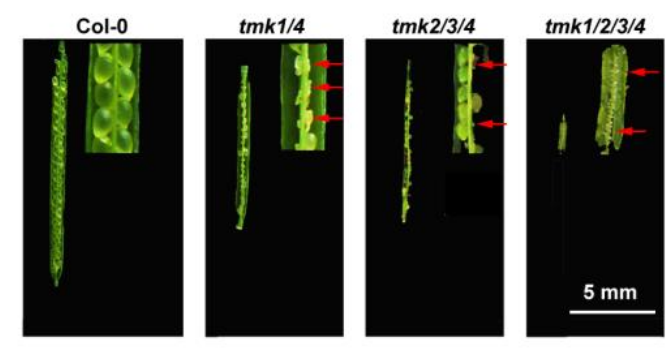

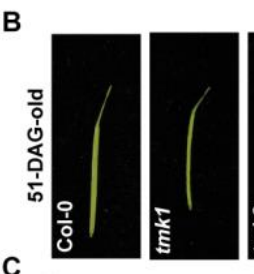
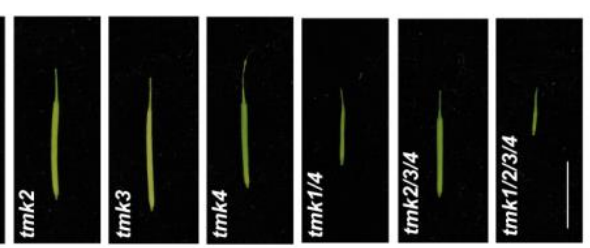

C

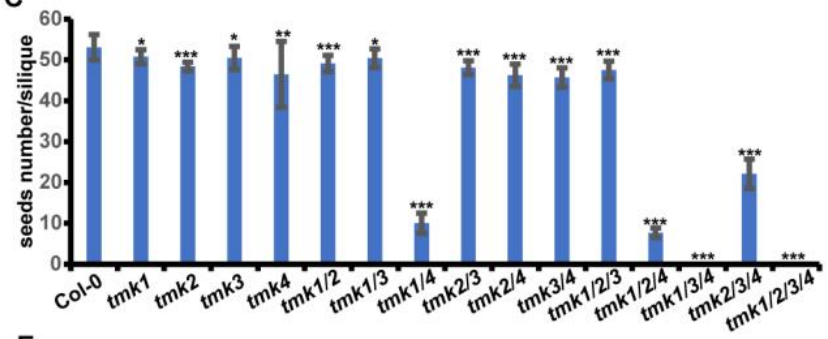

E
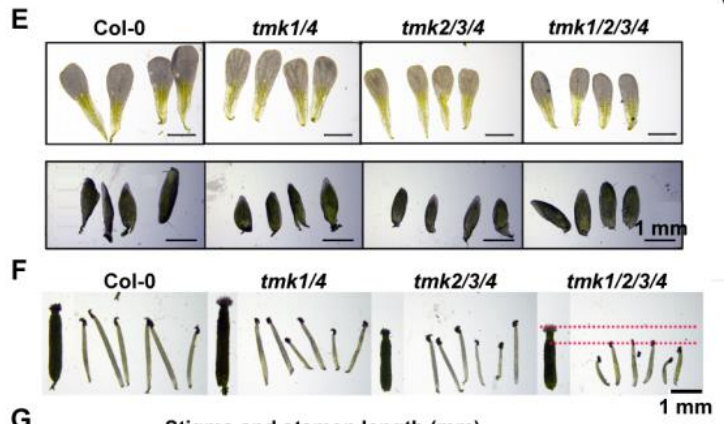

G
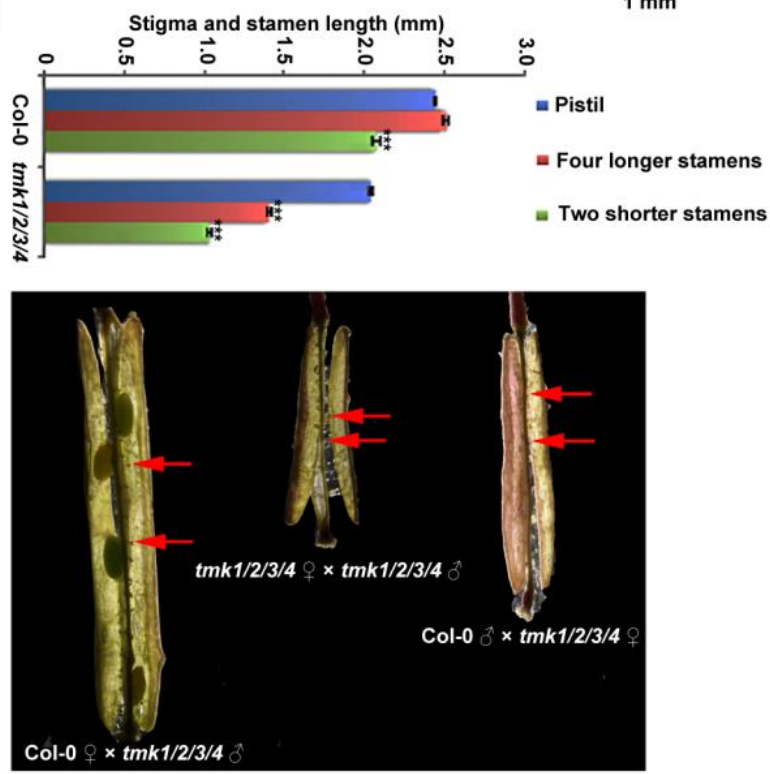

Figure 3. The novel tmk mutants displayed sexual growth defects.

(A) The shoots of 42-dag-old Col-0, tmk single mutants, tmk1/4, tmk2/3/4, and tmk1/2/3/4 mutants. Bars $=1 \mathrm{~cm}$.

(B) The siliques of 51-dag-old Col-0, tmk single mutants, tmk1/4, tmk2/3/4 and tmk1/2/3/4 mutants. Bar, $1 \mathrm{~cm}$.

(C) Quantitative analysis of the seeds number per silique in Col-0, tmk single mutants, tmk1/4, tmk2/3/4 
and tmk1/2/3/4 mutants.

(D) The intact flower of novel tmk mutants and Col-0 (45-d-old). Bar, $1 \mathrm{~mm}$.

(E) The sepals and petals comparison in 45-dag-old Col-0 and tmk1/4, tmk2/3/4 and tmk1/2/3/4 mutants. Bar, $1 \mathrm{~mm}$.

(F) The comparison of the stamens and stigma length of the novel $t m k 1 / 4, t m k 2 / 3 / 4$, and $t m k 1 / 2 / 3 / 4$ mutants and Col-0. The shortened length of stamen compared with stigma in tmk1/2/3/4 mutants was noted by red lines. Bar, $1 \mathrm{~mm}$.

(G) Quantitative analysis of stigma and stamen length in Col-0 and tmk1/2/3/4 mutant.

(H) Aborted seeds maturation in the novel tmk mutants. Bar, $5 \mathrm{~mm}$.

(I) The emasculated Col-0 flower that was pollinated with novel tmk1/2/3/4 mutant pollen developed only 3-5 mature seeds (the left panel); the emasculated novel tmk1/2/3/4 mutant flower that was pollinated with its own pollen and Col-0 pollen developed no seeds (the middle and right panels).

Note: t-test for comparison between tmk mutants and Col-0. Error bars denote \pm SD. “*”, “**”, and “***” indicate $\mathrm{p}<0.05, \mathrm{p}<0.01$, and $\mathrm{p}<0.001$, respectively. 


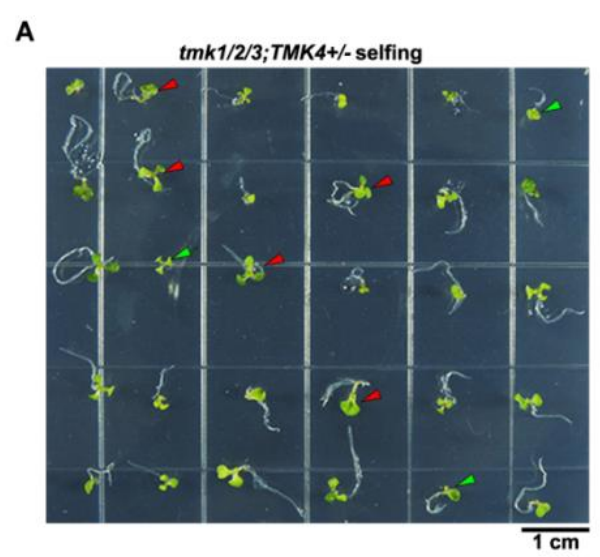

B

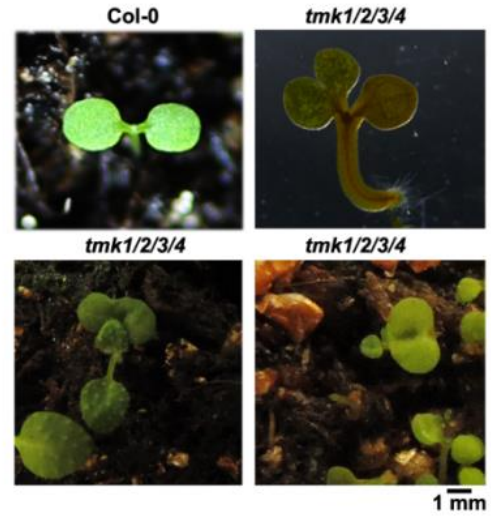

C

\begin{tabular}{|c|c|c|}
\hline phenotype of cotyledon & seeds of $t m k 1 / 2 / 3 ; T M K 4+/$ - selfing & ratio \\
\hline ungerminated & \multicolumn{2}{|c|}{$28 / 780(3.60 \%)$} \\
\hline \multirow{5}{*}{ aberrant cotyledon } & total & $25 / 780(3.20 \%)$ \\
\hline & $t m k 1 / 2 / 3 / 4$ & $3 / 780(0.38 \%)$ \\
\hline & tmk1/2/3;TMK4+/- & $15 / 780(1.92 \%)$ \\
\hline & $t m k 1 / 2 / 3$ & $2 / 780(0.26 \%)$ \\
\hline & genotyping failed & $5 / 780(0.64 \%)$ \\
\hline \multirow{5}{*}{ normal cotyledon } & total & $727 / 780(93.21 \%)$ \\
\hline & $t m k 1 / 2 / 3 / 4$ & $60 / 780(7.69 \%)$ \\
\hline & tmk1/2/3;TMK4+/- & $418 / 780(53.59 \%)$ \\
\hline & $t m k 1 / 2 / 3$ & $221 / 780(28.33 \%)$ \\
\hline & genotyping failed & $28 / 780(3.60 \%)$ \\
\hline
\end{tabular}

Figure 4. Phenotype analysis of tmk123;TMK4+/- selfing populations.

(A) Single, triple and swollen cotyledons of novel tmk mutants from the progenies of tmk1/2/3;tmk4+/selfing. Among the progenies of the tmk1/2/3;tmk4+/- selfing, the triple cotyledons with long root (shown by red arrows), single cotyledon with short root (shown by green arrows), and inflated cotyledons (shown by blue arrows) were observed. Bar, $1 \mathrm{~cm}$.

(B) Enlarged phenotype of the abnormal cotyledons of $t m k 1 / 2 / 3 / 4$ mutants grown in soil. Col-0 was used as a control. Bar, $1 \mathrm{~mm}$.

(C) Quantitative analysis of the abnormal cotyledon ratio of progenies of tmk1/2/3;tmk4+/- selfing. In 780 seedlings of tmk123; tmk4+/- selfing progenies, $3.60 \%$ seeds failed to germinate, while $3.20 \%$ showed abnormal cotyledon phenotype, which includes 0.38\% tmk123;tmk4-/-, 1.92\% tmk123;tmk4+/-, and $0.26 \%$ tmk $123 ;$ tmk $4+/+($ Chi-square test, $\mathrm{p}<0.01)$. 


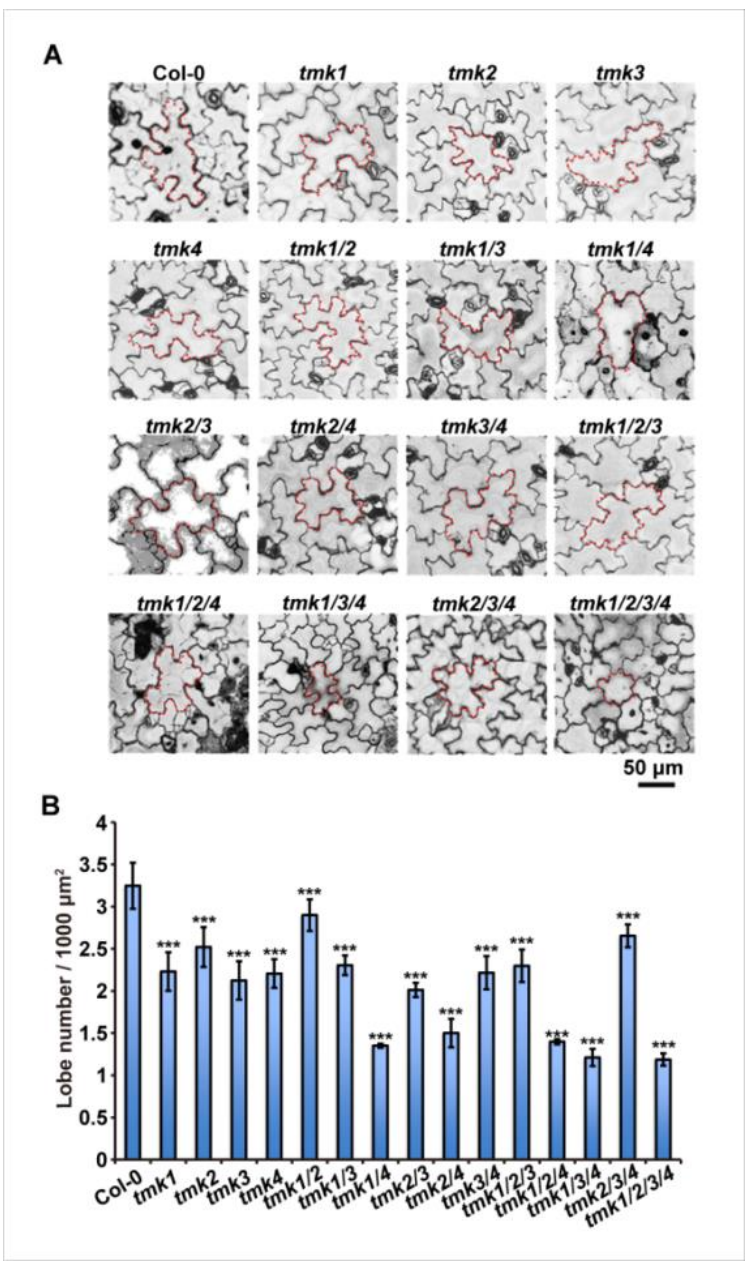

Figure 5. Pavement cell phenotype in Col- 0 and the tmk combination mutants.

(A) The pavement cell phenotype of Col-0 and all the generated tmk mutants. Bar, $50 \mu \mathrm{m}$.

(B) The lobe number per $\mu \mathrm{m}^{2 *} 1000$ of Col- 0 and all the generated tmk mutants.

Note: t-test for comparison between tmk mutants and Col-0. Error bars denote \pm SD. “*”, “**”, and “***” indicate $\mathrm{p}<0.05, \mathrm{p}<0.01$, and $\mathrm{p}<0.001$, respectively. 


\section{REFERENCES}

AZIZI P, RAFII MY, MAZIAH M, et al. 2015. Understanding the shoot apical meristem regulation: a study of the phytohormones, auxin and cytokinin, in rice. Mech Dev [J], 135: 1-15.

Benkova E, Michniewicz M, SAuER M, et al. 2003. Local, efflux-dependent auxin gradients as a common module for plant organ formation. Cell [J], 115: 591-602.

CAO M, CHEN R, LI P, et al. 2019. TMK1-mediated auxin signalling regulates differential growth of the apical hook. Nature [J], 568: 240-243.

Chang C, Schaller GE, Patterson SE, et al. 1992. The TMK1 gene from Arabidopsis codes for a protein with structural and biochemical characteristics of a receptor protein kinase. Plant Cell [J], 4: 1263-1271.

CSABA G 2015. Hormonal actions in the Protozoan stress: A review. Acta Microbiol Immunol Hung [J], 62: 331-339.

DAI N, WANG W, PATTERSON SE, et al. 2013. The TMK subfamily of receptor-like kinases in Arabidopsis display an essential role in growth and a reduced sensitivity to auxin. PLoS ONE [J], 8: e60990.

DEl Pozo JC, DiAz-Trivino S, Cisneros N, et al. 2006. The Balance between Cell Division and Endoreplication Depends on E2FC-DPB, Transcription Factors Regulated by the Ubiquitin SCF-SKP2A Pathway in Arabidopsis. Plant Cell [J], 18: 2224-2235.

Della Rovere F, FAtTorini L, D'ANGeli S, et al. 2013. Auxin and cytokinin control formation of the quiescent centre in the adventitious root apex of Arabidopsis. Ann Bot [J], 112: 1395-1407.

DHARMASIRI N, DHARMASIRI S, ESTELle M 2005. The F-box protein TIR1 is an auxin receptor. Nature [J], 435: 441-445.

Dubrovsky JG, SAUER M, NAPSUCialy-Mendivil S, et al. 2008. Auxin acts as a local morphogenetic trigger to specify lateral root founder cells. Proc Natl Acad Sci U S A [J], 105: 8790-8794.

GAO Y, ZHANG Y, ZHANG D, et al. 2015. Auxin binding protein 1 (ABP1) is not required for either auxin signaling orArabidopsisdevelopment. Proceedings of the National Academy of Sciences [J], 112: 2275-2280.

HERTEL R, THOMSON KS, RUSSO VE 1972. In-vitro auxin binding to particulate cell fractions from corn coleoptiles. Planta [J], 107: 325-340.

HUANG R, ZHENG R, HE J, et al. 2019. Noncanonical auxin signaling regulates cell division pattern during lateral root development. Proc Natl Acad Sci U S A [J], 116: 21285-21290.

JuRADo S, DiAZ-Trivino S, ABRAHAM Z, et al. 2008. SKP2A, an F-box protein that regulates cell division, is degraded via the ubiquitin pathway. Plant J [J], 53: 828-841.

KIM MH, KIM Y, KIM JW, et al. 2013. Identification of Arabidopsis BAK1-associating receptor-like kinase 1 (BARK1) and characterization of its gene expression and brassinosteroid-regulated root phenotypes. Plant Cell Physiol [J], 54: 1620-1634.

KLAMBT D 1990. A view about the function of auxin-binding proteins at plasma membranes. Plant Mol Biol [J], 14: 1045-1050.

LauX T, JURGEnS G 1997. Embryogenesis: A New Start in Life. Plant Cell [J], 9: 989-1000.

MANSFIEld G, G. BRIARTY L, ERNi S. Early embryogenesis in Arabidopsis thaliana. I. The mature embryo $\operatorname{sac}[\mathrm{C}] / /, 1991: 447-460.10 .1139 / \mathrm{b} 91-062$.

MoCKAITIS K, ESTELLE M 2008. Auxin receptors and plant development: a new signaling paradigm. Annu Rev Cell Dev Biol [J], 24: 55-80.

MOLler B, WeIJERS D 2009. Auxin control of embryo patterning. Cold Spring Harb Perspect Biol [J], 1: a001545.

Robert S, KLEINE-Vehn J, BARBEZ E, et al. 2010. ABP1 mediates auxin inhibition of clathrin-dependent endocytosis in Arabidopsis. Cell [J], 143: 111-121.

SANTNER A, ESTELLE M 2009. Recent advances and emerging trends in plant hormone signalling. Nature [J], 459: 1071-1078.

SASSI M, TRAAS J 2015. New insights in shoot apical meristem morphogenesis: Isotropy comes into play. Plant Signal Behav [J], 10: e1000150.

SCHALLER GE, BLEECKER AB 1993. Receptor-like kinase activity in membranes of Arabidopsis thaliana. FEBS Lett $[\mathrm{J}], 333: 306-310$.

SChWACHTJE J, KAROJET S, KUnZ S, et al. 2012. Plant-growth promoting effect of newly isolated rhizobacteria varies between two Arabidopsis ecotypes. Plant Signal Behav [J], 7: 623-627.

WANG Q, QIN G, CAO M, et al. 2020. A phosphorylation-based switch controls TAA1-mediated auxin biosynthesis in plants. Nat Commun [J], 11: 679.

XU T, DAI N, CHEN J, et al. 2014. Cell surface ABP1-TMK auxin-sensing complex activates ROP GTPase signaling. Science [J], 343: 1025-1028.

Xu T, WEN M, NAGAwA S, et al. 2010. Cell surface- and rho GTPase-based auxin signaling controls cellular interdigitation in Arabidopsis. Cell [J], 143: 99-110. 


\section{SUPPORTING INFORMATION}

Supplemental Table 1. List of primers used in this study.

\begin{tabular}{ccc}
\hline Primer name & Primer sequence (5'-3') & Primer usage \\
\hline TMK1-LP & CGCAATCTTCGTTCCATCGTGC & genotyping \\
TMK1-RP & TGATCCTAGAGTGAAGTCTTT & genotyping \\
TMK2-LP & TTTCTCATTCCATGTTCCAGC & genotyping \\
TMK2-RP & ACAAGGGAGAGTTGCATGATG & genotyping \\
TMK3-LP & TATCAACACCAACGGATTTGC & genotyping \\
TMK3-RP & TTGCCAGCTGTATTCACACAC & genotyping \\
TMK4-LP & TGCGATTGCTCAAAGAGGTCAGA & genotyping \\
TMK4-RP & ATACGCCGGTAAGTTCATTC & genotyping \\
LBb1.3 & ATTTTGCCGATTTCGGAAC & genotyping \\
GABIo8409 & ATATTGACCATCATACTCATTGC & genotyping \\
LB1 & GCCTTTTCAGAAATGGATAAATAGCCTTGCTTCC & genotyping \\
TMK1-Q-F & TCAAGAAAGCGATCGACACG & qRT-PCR \\
TMK1-Q-R & TTCTGATCCGACGGTTTCCA & qRT-PCR \\
TMK2-Q-F & TGCACCAGAGTATGCAGTGA & qRT-PCR \\
TMK2-Q-R & TGACCTCCATTGCTTCGTCT & qRT-PCR \\
TMK3-Q-F & AAGACCTGACATGGCTCACA & qRT-PCR \\
TMK3-Q-R & ACGAAGATCCCGAGTCATCC & qRT-PCR \\
TMK4-Q-F & AGGCACTCGACCAAACCTTA & qRT-PCR \\
TMK4-Q-R & ACGACGGTTTCCACTTCTCT & qRT-PCR \\
\hline
\end{tabular}

